\title{
MODELO DE GESTIÓN GAP Y LA MAXIMIZACIÓN EN EL NIVEL DE SATISFACCIÓN DEL CLIENTE: CASO RESTAURANTE CASA BLANCA.
}

\section{GAP MODEL OF SERVICE AND THE CUSTOMER SATISFACCIÓN: CASE OF STUDY CASA BLANCA RESTAURANT.}

Edison Fernando Chiriboga Cisneros, Mgs. Magíster en Administración de Empresas (Ecuador). Docente de la carrera de Hotelería y Turismo en la Universidad de Guayaquil, Ecuador.

edison.chiribogac@ug.edu.es

Mónica Angelina Pérez Zulueta, Mgs.

Magíster en Gestión Turística (Cuba). Docente tiempo completo de la carrera de Turismo y Hotelería de la Universidad Tecnológica ECOTEC, Ecuador. Docente a medio tiempo de la carrera de Hotelería y Turismo en la Universidad de Guayaquil, Ecuador. mperez@ecotec.edu.ec monica.perez@ug.edu.ec José Alonso Hidalgo León, Mgs. Magíster en Dirección y Gestión Turística (Ecuador). Docente de la carrera de Hotelería y Turismo en la Universidad de Guayaquil, Ecuador.

jose.hidalgol@ug.edu.ec Alexandra Fuentes Torres, Mgs.

Máster en Gestión y Administración de destinos y productos Turísticos (Ecuador). Docente a tiempo completo de la carrera de Hotelería y Turismo en la Universidad de Guayaquil, Ecuador.

\section{ARTÍCULO DE INVESTIGACIÓN}

Recibido: 23 de agosto de 2018.

Aceptado: 12 de noviembre de 2018. 


\section{RESUMEN}

El presente trabajo cumple con el propósito de medir el rango de satisfacción percibido por cliente. Se utilizaron métodos estadísticos para determinar la validez y confiabilidad del cuestionario, asimismo se utilizó el modelo gap con el objeto de detectar falencias, para así ofrecer un servicio de alta calidad. Los resultados mostraron que el rango de satisfacción es de 3,45 sobre 5 , y como conclusión se puede afirmar que los factores motivacionales, la capacitación, y un buen sistema de retroalimentación, maximizan el nivel de satisfacción del cliente.

Palabras clave: satisfacción del cliente, servicio, estrategias, modelo gap.

\section{ABSTRACT}

The present work fulfills the purpose of measuring the range of satisfaction perceived by the client. Statistical methods were used to determine the validity and reliability of the questionnaire, and the Gap model was used in order to detect flaws, in order to offer a high quality service. The results showed that the satisfaction rate is 3.45 out of 5 , and as a conclusion it can be affirmed that motivational factors, training, and a good feedback system, maximize the level of customer satisfaction.

Keywords: customer satisfaction; service; strategy, Gap model.

\section{INTRODUCCIÓN}

El turismo es considerado como una actividad económica, ya que genera riqueza y empleo, entre otros. La Organización Mundial de Turismo (OMT) señala que este sector, representa el $10 \%$ del PIB mundial, 7\% del comercio internacional y un puesto de trabajo por cada 11 existentes. Las estadísticas descritas, se replican en países con un alto potencial turístico, siendo éste el caso de Ecuador. De acuerdo al informe mensual del Ministerio de Turismo (MINTUR) de este país, para el año 2015, la contribución directa del turismo al PIB era del $1,6 \%$. Al igual que las empresas, los destinos turísticos han desarrollado estrategias para ser 
competitivos, enfatizando en aspectos de calidad técnica, imagen, servicios y precio, considerándose este último como el factor más utilizado por las organizaciones.

La competitividad ha sido estudiada por varios autores (Adam Smith 1776; David Ricardo 1817; Porter 1991; Bordas 1993; Esser 1994; Lever (1999), Begg 2002; Sobrino 2002), en este sentido (Hassan, 2000) (citado por lbáñez 2011) define a la competitividad turística como la capacidad de un destino para crear e integrar productos con valor añadido. Según lo mencionado, se debe inferir que un destino turístico es percibido como un todo por la demanda, es decir se deben utilizar todos los componentes ofertados por cada producto con la finalidad de incrementar la competitividad.

Para este estudio se decidió analizar una empresa de restauración debido a que contribuye a diferenciar un destino turístico con otro, (Colin Clark) (citado por Vargas y Aldana ,2011) el servicio de hospedaje y alimentación son cuasi domésticos por utilizar una casa, sin embargo, son considerados como un elemento económico. Además, se hace referencia a la clasificación del servicio propuesta por (Lovelock, 1984) (citado por Lloréns 1995) el cual sugiere dos categorías: según la naturaleza y al beneficiario del servicio, esta última presta beneficios a los consumidores, por ejemplo: restaurantes, transporte, salones de peluquería.

Es así que, constituye el propósito de este estudio la medición del nivel de satisfacción del cliente en el restaurante Casa Blanca. La percepción de satisfacción, se relaciona con la calidad del producto o servicio, y ésta a su vez, con el cumplimiento de los estándares o especificaciones del producto, lo cual se hace más difícil para las empresas de servicio debido a las características propias del sector, es decir su intangibilidad. En este contexto de múltiples variables condicionantes de la calidad y por ende de la satisfacción, se desarrolla esta investigación, tomando como principio básico que toda organización perteneciente a la industria del servicio debe estar orientada a satisfacer las expectativas del cliente.

Según Nayul Jodoy (2011) los compradores son más exigentes en la actualidad y tienen gran poder de compra, más aún exigen que el producto o servicio debe ser de calidad, por esta razón se necesita incorporar valor a través de: la conjunción de calidad en sus procesos y la atención al cliente, el primero Correía et, al (2012), menciona al proceso de manipulación y fabricación de alimentos como elementos primordiales para garantizar la calidad final de los productos ofrecidos, el segundo es una actividad desarrollada por las organizaciones con orientación a satisfacer necesidades. 
La calidad está relacionada con competitividad y debe estar en la planificación estratégica de la organización, más aún si se trata de productos gastronómicos, dado que la gastronomía es un referente cultural de cada país, además es considerado como una alternativa de desarrollo social y económico. Este estudio se estructuró de la siguiente manera: en la primera parte, se realiza una revisión de la bibliografía sobre servicio, calidad y calidad del servicio; segundo se mencionan los modelos de gestión de calidad; y por último se analizan los resultados obtenidos después de la presentación de los métodos de investigación aplicados.

Este trabajo presenta un alcance descriptivo, el cual permitió conocer las causas que producen quejas sobre los servicios ofertados del restaurante, de tal forma es importante identificar los modelos que miden el nivel de satisfacción al cliente y que han sido utilizados en diferentes contextos. Para este estudio se utilizó el modelo Gap, siendo éste la base de otros modelos de gestión de calidad propuestos.

Para la elección de la muestra se determinó las características de la misma, estando compuesta por aquellos sujetos que visitaron el restaurante, el objeto de estudio se encuentra localizado en la provincia de Santa Elena, según los datos del MINTUR, (2015) este lugar tiene registrado a 490 establecimientos (restaurantes), además la comuna antes mencionada es la playa más reconocida a nivel internacional.

\section{REVISIÓN TEÓRICA}

\subsection{Servicio y Calidad}

Al momento de teorizar sobre "calidad del servicio", se debe mencionar que "servicio y calidad" han sido aspectos estudiados por separado, es más poseen un acopio de definiciones, discrepancias, e investigaciones empíricas en diferentes contextos.

Es importante recalcar que las organizaciones, debido a los efectos de la globalización plantearon al servicio como una variable de competitividad, Parazuraman, et al. (1994) menciona que desde 1970 pasó de una economía de producción a la de servicios, y que comparte características únicas, por ejemplo: intangibilidad, no diferenciación entre producción y entrega y la inseparabilidad de la producción y el consumo. 
Se pone de manifiesto en esta investigación la lista de los principales autores que han conceptualizado al servicio desde 1983 hasta el 2011; (Gaither 1983 p. 183; Larrea p. 1986; Stanton 1986 p 554; kotler 1987 p 514; Langeard 1989; Berry benet y brown 1989; Lovelock 1990; Horovitz 1990 p. 2; Rosander 1992; Peel 1993; Payner 1993; Lamata 1994; Albretech,1994, p.116; Fisher y Navarro,1994, p. 185; Berry 1995; Colunga, 1995, p. 25; Thomas 1995; Lobos 1996; Juran 1996; Tschohl 1997 p 11; Larousse 2005; Vargas et al. (2011).

Se ha tomado en cuenta el concepto generado por Vargas et al. (2011), el cual expresa que servicio es el conjunto de actividades diseñadas para construir procesos que conduzcan a incrementar la satisfacción de las necesidades, deseos y expectativas de quien lo necesite.

Lo anterior ratifica al consumidor como juez sobre el servicio consumido, más aún los resultados obtenidos en diferentes contextos mostraron que existe satisfacción cuando se superan las expectativas. (Tse y Wilton, 1988) (citado por Sánchez, 2009) señalaron que los resultados que los clientes perciben directa e indirectamente con la satisfacción, van más allá del proceso de confirmación de expectativas.

El término expectativa apareció por primera vez en aspectos motivacionales de la conducta, según (Navas, 1990) (citado por Peralta, 2006) fue utilizado en la psicología por teóricos como Edward Tolman y Kurt Lewin, así mismo existe distinción entre expectativas cognitivas y afectivas, la primera es de carácter general asociada a las creencias anticipatorias del servicio y su calidad, mientras que la segunda, estaría directamente asociada a la experiencia afectiva y su confirmación, que conlleva al sentimiento de satisfacción o insatisfacción.(Peralta, 2006).

Oliver y Miller (1997) clasifican respectivamente a la expectativa según el nivel deseado y la abstracción; Parazurnam et al. (1985) menciona que existe un rango entre el nivel deseado y adecuado, es más, existen varios niveles como: deseado, nivel esperado, mínimo aceptable y merecido, pero todo esto depende del nivel de motivación que tienen los usuarios.

La calidad asociada al servicio ofrece una expectativa cognitiva, es decir, el servicio de calidad provoca el desarrollo de satisfacción al cliente, para afirmar lo aunado a lo anterior (Duque, 2005) (citado por Moliner et al., 2015) señala que la calidad se basa en dos tendencias: objetiva y subjetiva, la primera tiene que ver con la producción y se enfoca en la 
perspectiva del productor, la segunda tiene que ver con la perspectiva del consumidor y se enfoca en la satisfacción de las necesidades.

Es así que esta variable (calidad), ha contribuido en la formación de varias escuelas, por ejemplo en el campo de la administración: Fredrick Taylor - Henri Fayol, teoría científica; Iton Mayo - Fritz Roethlisbergef, escuela de relaciones humanas; Max Weber, escuela burocrática; Herbert Simon - Russell Ackoff, escuela cuantitativa; Kliksberg - Joseph McGuire - J. Pfiffner, escuela de sistemas sociales; Alvin Brown - Ernest Dale - Peter Drucker - William Newman - Henry Mintzberg, escuela neoclásica; Ledwing Von Bertalamfly; Jay Foster; Melese; Rcihard Johnson; Fremont kast; james Rosen; zweig; escuela de sistemas; Joan Woodward- Tom Burns - Gm Stalker, escuela contingente; Gronroos, escuela nórdica; Parazurnam, escuela americana.

De acuerdo, con lo anteriormente expuesto se puede señalar que el estudio exhaustivo de esta variable ha contribuido en la formación de varias teorías, por ejemplo: Edwars Deming, teoría de Deming; Joseph Juran, teoría de la planificación de la calidad; Karow Ishikawa, teoría de la Calidad Total; Armand Feigenbaum, Teoría de calidad basada en la administración; Genichi Taguchi, ingeniería de la calidad; Crosby, teoría de lo cero errores; Shigeo Shingo, teoría de poke yoke; Oakland Jhon, teoría contemporánea; Shigero Mizuno, teoría de la política de calidad; Eliyahu Goldratt, teoría de las restricciones; Tom Peters- Robert Waterman-Nancy Austin, teoría de la excelencia; karl Albretch, gerencia del valor al cliente; Jacques Horovitz, teoría de la diferenciación en el servicio; Christhian Groonros, teoría de la mercadotecnia; James Heskett, elementos básicos integradores. Así pues, la calidad ha generado diferentes criterios y conceptualizaciones desde la década de 1980.

\subsection{Calidad del servicio}

La Calidad del servicio ha sido adoptado por organizaciones debido a muchos de los beneficios que ofrece, por ejemplo: mejora los procesos, diferenciación con la competencia, penetración de mercado, productividad, costos, motivación al personal, y otros, es decir lograr mayor eficiencia para ofrecer un mejor servicio.

Existen varios autores que han conceptualizado el término "calidad del servicio ", como: Oliver,1980; Lewis y Booms, 1983; Parazurnam et al, 1985; Cronin y Tailor, 1992,194; (Grönroos, 1994); alet, 2000; Chao, 2008; Wang, 2009; Vargas, 2011; Torres, 2012, Ruiz, 
2012). La definición más utilizada es de Parazurnam et al. (1984) el cual expresa que la calidad del servicio es la discrepancia entre las expectativas de los consumidores sobre el servicio que van a recibir y sus percepciones sobre el servicio prestado por la empresa.

La calidad del servicio ha transcendido, incluso se ha comprobado con evidencias empíricas sobre la relación directa o indirecta de este término con otras variables, por ejemplo, Cronin et al. 2000; Brady et al., 2005 (citado por Moliner et al. 2015) expresa que la variable calidadvalor(social y percibido)-satisfacción afecta sobre la lealtad; Wang, (2009) (citado por Mondo, 2016) señala que la calidad del servicio y la satisfacción inciden de manera directa en la experiencia así como el impacto en la tecnología y la reputación, además la satisfacción de las necesidades-crea lealtad y provoca la comunicación boca a boca (positiva o negativa); García (2016) señalan que la gestión de la calidad con la innovación es positiva; es decir, calidad es considerado por varios autores como un término multidimensional.

Gestionar la calidad del servicio es un factor determinante clave para garantizar el buen desempeño empresarial y que a través de ésta se puede aumentar la capacidad competitiva y el crecimiento económico. (Camisón, 1996; Devlin y Dong, 1994; Sureshghandar, Chandrasekharan y Anantharaman, 2002; Corbett, 1994, Kim 1995).

\subsection{Medición de la satisfacción al cliente}

Así, la evaluación de la calidad del servicio está encabezada por la escuela nórdica y americana, la primera está dirigida por varios investigadores como: Grönroos $(1982,1988)$ y Lehtinen y Lehtinen (1991). Las investigaciones realizadas por estos autores se basan en la tridimensionalidad de la variable calidad.

La segunda la encabeza Parasuraman, Zeithaml y Berry $(1985,1988)$, sus investigaciones se basan en plantear el modelo SERVQUAL como un instrumento que permite medir la satisfacción del servicio mediante la diferencia entre las expectativas y la percepción del servicio, así mismo, ha sido el modelo con mayores estudios consecutivos.

En el contexto turístico este modelo ha sido utilizado en: hoteles de sol y playa por Getty Thompson, 1994; Ruiz Vásquez y Diaz,1995; Falces et al, 1999; turismo rural por Ruiz, Vásquez y Diaz, 1995); agencia de viajes por Bigné, 1999; transporte aéreo por Robledo 1997; áreas protegidas por Zamora, León, \& Andrades, 2009; educación por Duque y Chaparro, 
2012; transporte terrestre por Gil y Berenguer, 2015; servicios turísticos por Betencourt, (2016).

Sin embargo, varios autores han sido detractores de Parasuraman et al, y muchos han seguido sus investigaciones, por consiguiente en muchas estudios se han desarrollado varios modelos para medir el nivel de la satisfacción del cliente, por ejemplo Oliver, 1980 confirmó la teoría de la desconfirmación; Groonross, 1984 modelo de la calidad del servicio; Parasuraman, et al, 1985 modelo SERVQUAL; Eiglier y Langeard, 1989 Modelo de Servucción; Cronin y Taylor, 1992 modelo SERVPERF; Teas, 1994 Modelo de Desempeño Evaluado; Rust y Oliver, 1994 Modelo de los Tres Componentes; Dabholkar, Thorpe y Rentz, 1996 Escala Multinivel de Calidad del Servicio; Philip y Hazlett, 1997 Modelo P-C- P; Brady y Cronin, 2001 Modelo Jerárquico Multidimensional; Akbaba, 2006 Técnica del Factor Incidente Crítico.

En el ámbito turístico ha sido utilizado por Mondo, 2014 el modelo Tourqual, LODGSERV (Knutson et al. 1990) para la industria del alojamiento, Giqual del alojamiento, GIQUAL (Tsoukatos et al., 2004) para la industria de seguros; Airqual para el sector de aerolíneas; DINESERV (Stevens et al., 1995) para la industria de restaurantes); LODQUAL para la industria aplicado a medios de alojamiento (Getty\& Thompson, 1994); HOTELQUAL para servicios de alojamiento (Falces et al, 1999). SERVPERF que tiene el mismo concepto del SERVQUAL.

\subsection{Modelo Gap}

Oliver, 1980 formuló la teoría de la desconfirmación, y menciona que este se basa en las percepciones, así mismo Parazuraman et al, (1985) menciona que las percepciones son las creencias de los consumidores sobre el servicio recibido, este se basó en la teoría del consumidor. Sobre esta base Parazuraman et al. (1985) desarrolló el modelo servqual para medir la percepción del consumidor entre lo esperado y lo recibido.

Para evaluar la calidad del servicio se han planteado varias dimensiones, por ejemplo, Groonros (1982-1984); Leithenen (1991); Lewis (1993) plantean la calidad técnica y funcional; Goodman, Marra y Birgham (1986) identifican las dimensiones claves y su impacto en la satisfacción del cliente; Parazuraman et al (1985) señala tres dimensiones: física, corporativa e interactiva, así mismo años después se basaron en diez dimensiones; Zeithaml, y Biener 
,(2001) indican cinco dimensiones: confianza, responsabilidad, empatía, seguridad y tangibilidad.

El modelo SERVQUAL se basa en cinco dimensiones propuestas por Parazuraman et al, (1985) las cuales son fiabilidad, garantía, capacidad de respuesta, tangibilidad y empatía, compuesta por 22 preguntas que evalúan las expectativas y el valor percibido, sin embargo, existen cinco desajustes o "gaps" en el proceso de desarrollo, estos influyen en la percepción del cliente, es decir los cinco vacíos o "gaps" que influyen en la calidad del servicio.

En la figura 1, se puede concluir que el gap \# 1 es la discrepancia entre las expectativas del cliente y las percepciones de la empresa; gap \# 2 es la discrepancia entre la percepción de los directivos y las expectativas de los clientes; gap \# 3 es la discrepancia entre las especificaciones de calidad y el servicio realmente ofrecido; gap \# 4 discrepancia entre el servicio real y lo que se comunica a los clientes sobre él; gap \# 5 diferencia de las expectativas y percepciones del cliente.

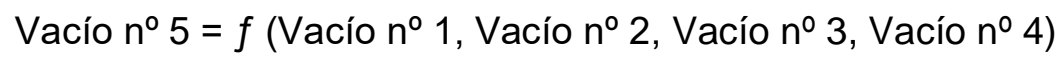

Figura 1. Modelo gap propuesto

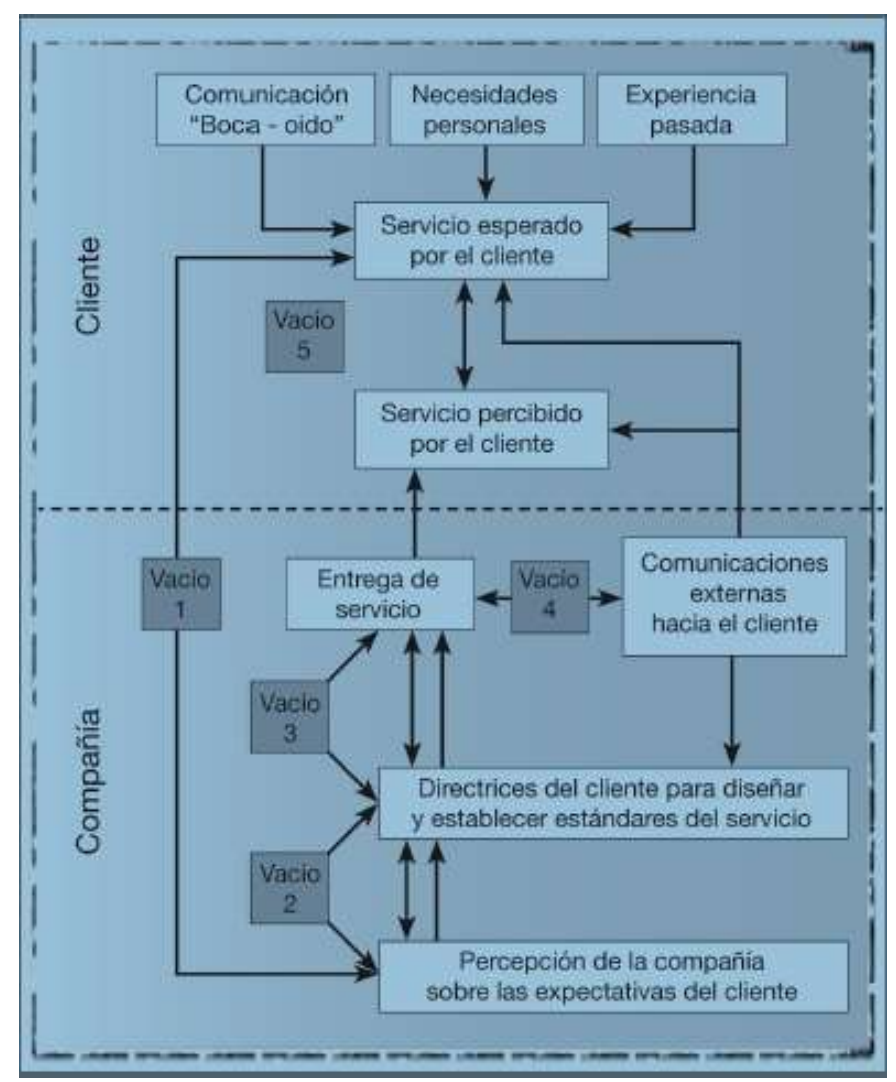

Fuente: Parazuraman et al. (1985, p.16). 


\section{MATERIALES Y MÉTODOS}

El enfoque de este trabajo fue cuantitativo, empírico y bibliográfico, debido a que fue necesario recolectar datos confiables y así obtener el tamaño muestral que permita cumplir con el objetivo principal de la investigación, es así, que el muestreo probabilístico aleatorio simple, en el cual (Hernández, et. al 2014) lo define como un subgrupo de la población en el que todos los elementos tienen la misma posibilidad de ser elegidos, servirán de base para tomar decisiones en la medición de la calidad del servicio del objeto de estudio.

\subsection{Participantes}

La población considerada en esta investigación fue de 10.400 visitantes entre turistas nacionales y extranjeros que acudieron al restaurante "La Casa Blanca" en la comunidad Montañita dese el mes de enero de 2017 a julio del mismo año, además se realizó una entrevista al administrador del restaurante para conocer sobre la importancia de la implementación de un sistema de gestión de calidad que constó de cinco preguntas.

Para determinar la muestra de esta investigación se utilizó el programa Decision analysis Stats 2.0, el cual estima un porcentaje de error máximo admisible del 10\%; porcentaje de éxito estimado del $50 \%$; y un nivel de confianza del $95 \%$.

El resultado del tamaño muestral para la presente investigación fue de 95 clientes que se necesitan para representar al total de visitantes en el restaurante Casa Blanca.

\subsection{Procedimiento}

La recolección de los datos fue en el lugar donde se presta el servicio, y se obtuvo el permiso necesario por parte del administrador de dicho establecimiento. Todos los visitantes que consumieron en el restaurante contestaron el cuestionario de manera voluntaria y anónima, este instrumento constó de 16 preguntas adaptadas según el modelo de gestión Gap, además se realizaron dos preguntas adicionales para conocer la percepción de satisfacción con el servicio y la posibilidad de comunicación boca-oído.

\subsection{Instrumentos}

Los ítems están basados en 3 dimensiones: infraestructura, talento humano y producto, asimismo, fueron valorados según la escala de Likert de 1 al 5, sin embargo, cada una tiene diferentes escalas, a continuación, se detallan los parámetros: 
La percepción del cliente basado en la "infraestructura" está valorada de 1 al cinco y está según la escala: muy atractiva (5); atractiva (4); aceptable (3); poco atractiva (2) y nada atractiva (1), sin embargo, en la pregunta de aseo, perteneciente a esta dimensión fue medida según la escala: Muy aseado (5); aseado (4); aceptable (3); desaseada (2) y muy desaseada (1).

La percepción del cliente basado en el "talento humano" está valorada de 1 al cinco y está según la escala: excelente (5); muy buena (4); buena (3); regular (2) y mala (1).

La percepción del cliente basado en el "producto" está valorada de 1 al cinco y está según la escala: excelente (5); muy buena (4); buena (3); regular (2) y mala (1).

\subsection{Análisis estadístico}

El cuestionario debe ser viable y confiable, es así que (Hernández, et. al, 2014) señala que en muchos de los casos el instrumento es viable pero no confiable y si es así los resultados de la investigación no deben tomarse en serio. La confiabilidad según (Chavez, 2001, p 75) (citado por Árraga y Sánchez, 2012, p 383) se define como el "grado de congruencia con que se realiza la medición de la variable.

Se calculó la confiabilidad del instrumento mediante el alfa de Cronbach, su característica principal es que el resultado debe mantenerse en un rango de 0 a 1 , entre más cercano al 1 más confiable es el cuestionario, es así que este método estadístico mide la confiabilidad en base a dos números: el número de ítems y la varianza total de la prueba debido a la covarianza total de los ítems.

$$
\frac{k}{k 1} * 1 \frac{S i^{2}}{S^{2} n u m}
$$

K: es el número de ítems de la prueba

$\mathrm{S}^{2}$ : es la varianza de los ítems (desde 1...núm.)

$\mathrm{S}^{2}$ : es la varianza total de los ítems.

El análisis factorial (prueba de validez), es la ratificación de las calificaciones de una prueba y las variables se articulan en cierto patrón predicho por una teoría, con el propósito de generar una descripción de un conjunto amplio y complejo de datos y así presenta como un punto 
inicial, la matriz de correlación y una tabla completa de inter-correlaciones entre todas las variables (Árraga \& Sánchez, 2012).

El análisis factorial comparte tres puntualizaciones representativas, primero la medida de adecuación muestral Kaiser-Meyer- Olkin (KMO), que toma valores entre 0 y 1, aunque si está por encima de 0,6, indica que se puede utilizar el análisis factorial. Véase lo siguiente Valor de:

$\mathrm{KMO}$ menor que 0.5 . se considera muy malo

$\mathrm{KMO}$ entre 0.5 y 0.59 ; se considera malo

$\mathrm{KMO}$ entre 0.6 y 0.69 : se considera regular

$\mathrm{KMO}$ entre 0.7 y 0.79 : se considera aceptable

$\mathrm{KMO}$ entre 0.80 y 0.89 : se considera bueno

$\mathrm{KMO}$ entre 0.90 y 1 . Se considera excelente.

Segundo la prueba de esfericidad de Bartlett considerada como una matriz de identidad, la cual su análisis indica la relación entre variables y su rango es de 0 a 1. Por último, se plantea la tabla de comunalidad que explica cómo una variable se relaciona con el resto de variables y así obtener un factor en común.

Tabla 1. Resultados correspondientes al coeficiente del alfa de Cronbach.

\begin{tabular}{lcccc}
\hline \multirow{2}{*}{ Dimensiones } & \multirow{2}{*}{ Alfa de cronbach } & \multicolumn{2}{c}{ valores } \\
\cline { 3 - 5 } & & válidos & \multicolumn{2}{c}{ excluidos } \\
\hline dimensión 1 & 0,987 & 8 & 0 \\
dimensión 2 & 0,947 & 3 & 0 \\
dimensión 3 & 0,96 & 5 & 0 \\
\hline
\end{tabular}

Fuente: Elaboración propia a partir de datos tomados por el software SPSS 21v.

A partir de los datos generados por el software SPSS v.21 la media del alfa de Cronbach para las tres dimensiones fue de 0.990 cercano a 1 , lo que conlleva a garantizar el cuestionario, así mismo se observa que cada dimensión se encuentra en un rango de excelente. 
Tabla 2. Resultados correspondientes del KMO y Bartlett.

\begin{tabular}{lccc}
\cline { 2 - 4 } & \multicolumn{3}{c}{ Dimensiones } \\
& dimensión 1 & dimensión 2 & dimensión 3 \\
\hline \multicolumn{1}{c}{$\begin{array}{c}\text { medida kaiser-Meyer-Olkin (Kmo) } \\
\text { chi cuadrado }\end{array}$} & 0,932 & 0,739 & 0,847 \\
prueba de esferidad de Bartlett & 1620,144 & 299,853 & 651,651 \\
\hline gl & 28 & 3 & 10 \\
significancia & 0,000 & 0,000 & 0,000 \\
\hline
\end{tabular}

Fuente: Elaboración propia a partir de datos obtenidos por el software SPSS v.21.

A partir de estos datos según la medida $\mathrm{KMO}$, se puede concluir que la dimensión uno es considerada excelente; la segunda es considerada aceptable y la última es considerada buena. Asimismo, el grado de significancia es menor a 0,5 por lo que cada dimensión es válida.

Tabla 3. Comunalidades para cada dimensión.

\begin{tabular}{|c|c|c|c|}
\hline & Atributos que pertenecen a las dimensiones (comunalidades) & inicial & extracción \\
\hline \multirow{10}{*}{ DIM 1} & $\begin{array}{l}\text { ¿Cómo percibió la infraestructura del Restaurante La Casa Blanca? } \\
\text { ¿Qué impresión le dejó el aseo yla higiene en el Restaurante La }\end{array}$ & 1,000 & 0,953 \\
\hline & Casa Blanca? & 1,000 & 0,951 \\
\hline & ¿Observó moscas, insectos y/o roedores en el Restaurante La Casa & & \\
\hline & Blanca? & 1,000 & 0,848 \\
\hline & ¿Había tachos de basura muy aseados en el Restaurante La Casa & & \\
\hline & Blanca? & 1,000 & 0,943 \\
\hline & ¿Cómo encontró las paredes del Restaurante La Casa Blanca? & 1,000 & 0,939 \\
\hline & ¿Cómo encontró el techo del Restaurante La Casa Blanca? & 1,000 & 0,927 \\
\hline & $\begin{array}{l}\text { ¿Cómo encontró las sillas y mesas del Restaurante La Casa Blanca? } \\
\text { ¿Cómo observó el área de la cocina del Restaurante La Casa }\end{array}$ & 1,000 & 0,916 \\
\hline & Blanca? & 1,000 & 0,918 \\
\hline \multirow{3}{*}{ DIM 2} & $\begin{array}{l}\text { ¿Cómo percibió la atención del personal del Restaurante La Casa } \\
\text { Blanca? }\end{array}$ & 1,000 & 0,947 \\
\hline & $\begin{array}{l}\text { ¿Cómo encontró la vestimenta del personal del Restaurante La } \\
\text { Casa Blanca? }\end{array}$ & 1,000 & 0,883 \\
\hline & $\begin{array}{l}\text { ¿Qué opinión le merece la rapidez en la atención en el } \\
\text { Restaurante La Casa Blanca? }\end{array}$ & 1000 & 0.909 \\
\hline \multirow{7}{*}{ DIM 3} & ¿Qué opinión le merece la carta de menús en el Restaurante La & & \\
\hline & Casa Blanca? & 1,000 & 0,943 \\
\hline & $\begin{array}{l}\text { ¿Qué opina acerca de la comunicación e información con el } \\
\text { personal del Restaurante La Casa Blanca? }\end{array}$ & 1,000 & 0,81 \\
\hline & $\begin{array}{c}\text { En su opinión ¿Qué tan bueno es el alimento que le ofreció el } \\
\text { Restaurante La Casa Blanca? }\end{array}$ & 1,000 & 0,908 \\
\hline & En su opinión ¿Qué tan grasoso es el alimento que le ofreció el & & \\
\hline & Restaurante La Casa Blanca? & 1,000 & 0,845 \\
\hline & $\begin{array}{l}\text { ¿Qué tan aseado se encontraban los platos ylas cucharas que le } \\
\text { ofreció el Restaurante La Casa Blanca? }\end{array}$ & 1,000 & 0,901 \\
\hline
\end{tabular}

Fuente: Elaboración propia a partir de datos obtenidos por el software SPSS v.21. 
A partir de estos datos se puede observar que todos los valores son superiores a 0,400 por lo que se concluye que el cuestionario es fiable y tiene validez.

\section{RESULTADOS}

Para el cálculo del rango de satisfacción se elaboró un cuadro de ponderaciones compuesta por los atributos pertenecientes a las tres dimensiones investigadas, además incluye la valoración según la escala de Likert de 1 a 5 , en donde 5 corresponde a excelente y 1 es malo, así mismo se incluye la frecuencia y la ponderación de cada atributo.

El rango de satisfacción del cliente es de 3,45 sobre 5, lo que corresponde un $69 \%$ de aceptación de los clientes al servicio ofrecido por el restaurante Casa Blanca, y así encontrarse en el rango de 60-80\% considerado como "Bueno".

\subsection{Rango de calificación sensorial por dimensión}

En la dimensión 1, compuesta por ocho atributos se puede observar que los números 2, 6 y 7 son las peores percibidas por el cliente, debido a que se encuentran por debajo de la media $(3,58)$, es así necesario mejorar la atención que ofrece el personal, el desempeño, la comunicación, tiempo en que se presta la atención, rapidez en el servicio, responsabilidad, puntualidad en la prestación del servicio, todos estos aspectos colaboran para las necesidades y requerimientos de los clientes.

Tabla 4. Dimensión \# 1

\begin{tabular}{|c|c|c|c|}
\hline Atributos & Ponderación total & $\begin{array}{l}\text { Tamaño de la } \\
\text { muestra }\end{array}$ & Rango obtenido \\
\hline ¿Cómo percibió la infraestructura del Restaurante La Casa Blanca? & 337 & 95 & 3,55 \\
\hline $\begin{array}{l}\text { ¿Qué impresión le dejó el aseo y la higiene en el Restaurante La } \\
\text { Casa Blanca? }\end{array}$ & 311 & 95 & 3,27 \\
\hline ¿Observó moscas, insectos y/o roedores en el Restaurante La Casa & & & \\
\hline Blanca? & 394 & 95 & 4,15 \\
\hline ¿Había tachos de basura muy aseados en el Restaurante La Casa & & & \\
\hline Blanca? & 343 & 95 & 3,61 \\
\hline ¿Cómo encontró las paredes del Restaurante La Casa Blanca? & 362 & 95 & 3,81 \\
\hline ¿Cómo encontró el techo del Restaurante La Casa Blanca? & 311 & 95 & 3,27 \\
\hline ¿Cómo encontró las sillas y mesas del Restaurante La Casa Blanca? & 327 & 95 & 3,44 \\
\hline ¿Cómo observó el área de la cocina del Restaurante La Casa Blanca? & 335 & 95 & 3,53 \\
\hline PROMEDIO & 340 & 95 & 3,58 \\
\hline
\end{tabular}

Fuente: Elaboración propia a partir de datos obtenidos por el software SPSS v.21 
Gráfico 1. Percepción del cliente.

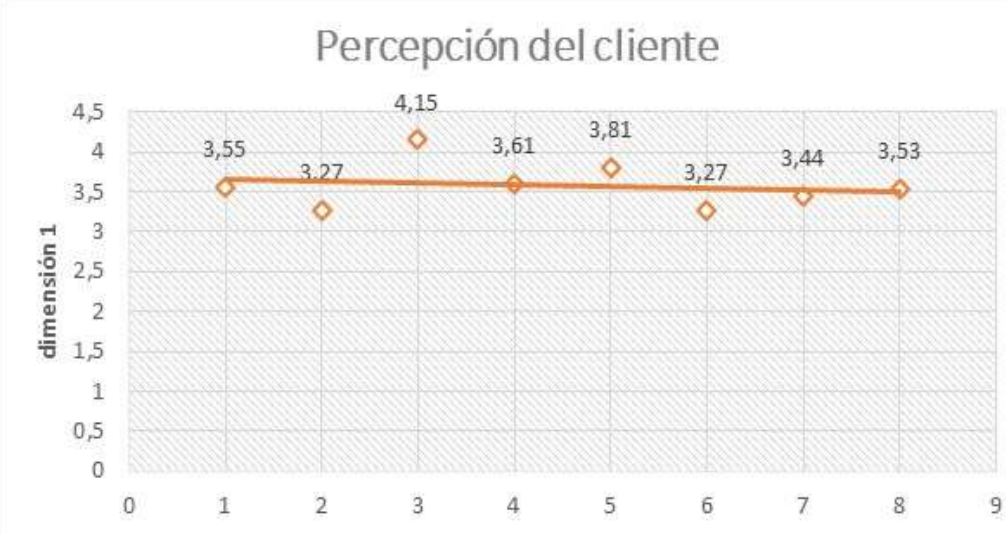

Fuente: Elaboración propia.

En la dimensión 2, compuesta por tres atributos se puede observar que el 1 y 3 son los peores percibidos por el cliente, debido a que se encuentran por debajo de la media $(2,83)$.

Tabla \# 5. Dimensión \# 2.

\begin{tabular}{|c|c|c|c|c|}
\hline \multirow{8}{*}{ DIM 2} & Atributos & Ponderación total & $\begin{array}{l}\text { Tamaño de la } \\
\text { muestra }\end{array}$ & Rango obtenido \\
\hline & \multicolumn{4}{|l|}{ ¿Cómo percibió la atención del personal del Restaurante La Casa } \\
\hline & Blanca? & 205 & 95 & 2,16 \\
\hline & \multicolumn{4}{|l|}{ ¿Cómo encontró la vestimenta del personal del Restaurante La Casa } \\
\hline & Blanca? & 372 & 95 & 3,92 \\
\hline & \multicolumn{4}{|l|}{ ¿Qué opinión le merece la rapidez en la atención en el Restaurante } \\
\hline & La Casa Blanca? & 229 & 95 & 2,41 \\
\hline & PROMEDIO & 268,67 & 95 & 2,83 \\
\hline
\end{tabular}

Fuente: Elaboración propia a partir de datos obtenidos por el software SPSS v.21.

Gráfico 2. Percepción del cliente.

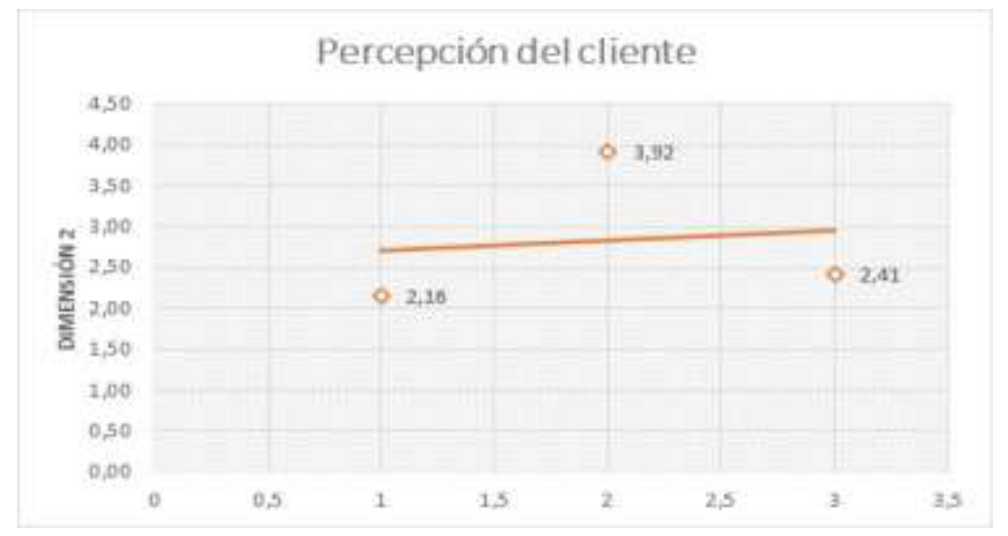

Fuente: Elaboración propia. 
En la dimensión 3, compuesta por cinco atributos se puede observar que el 2 es el peor percibido por el cliente, debido a que se encuentra por debajo de la media $(3,62)$.

Tabla \# 6. Dimensión \# 3.

\begin{tabular}{|c|c|c|c|}
\hline Atributos & Ponderación total & $\begin{array}{l}\text { Tamaño de la } \\
\text { muestra }\end{array}$ & Rango obtenido \\
\hline \multicolumn{4}{|l|}{ ¿Qué opinión le merece la carta de menús en el Restaurante La Casa } \\
\hline Blanca? & 371 & 95 & 3,91 \\
\hline ¿Qué opina acerca de la comunicación e información con el personal & & & \\
\hline del Restaurante La Casa Blanca? & 213 & 95 & 2,24 \\
\hline En su opinión ¿Qué tan bueno es el alimento que le ofreció el & & & \\
\hline Restaurante La Casa Blanca? & 350 & 95 & 3,68 \\
\hline En su opinión ¿Qué tan grasoso es el alimento que le ofreció el & & & \\
\hline Restaurante La Casa Blanca? & 397 & 95 & 4,18 \\
\hline $\begin{array}{l}\text { ¿Qué tan aseado se encontraban los platos y las cucharas que le } \\
\text { ofreció el Restaurante La Casa Blanca? }\end{array}$ & 390 & 95 & 4,11 \\
\hline PROMEDIO & 344,2 & 95 & 3,62 \\
\hline
\end{tabular}

Fuente: Elaboración propia a partir de datos obtenidos por el software spss v.21.

Gráfico \# 3. Percepción del cliente.

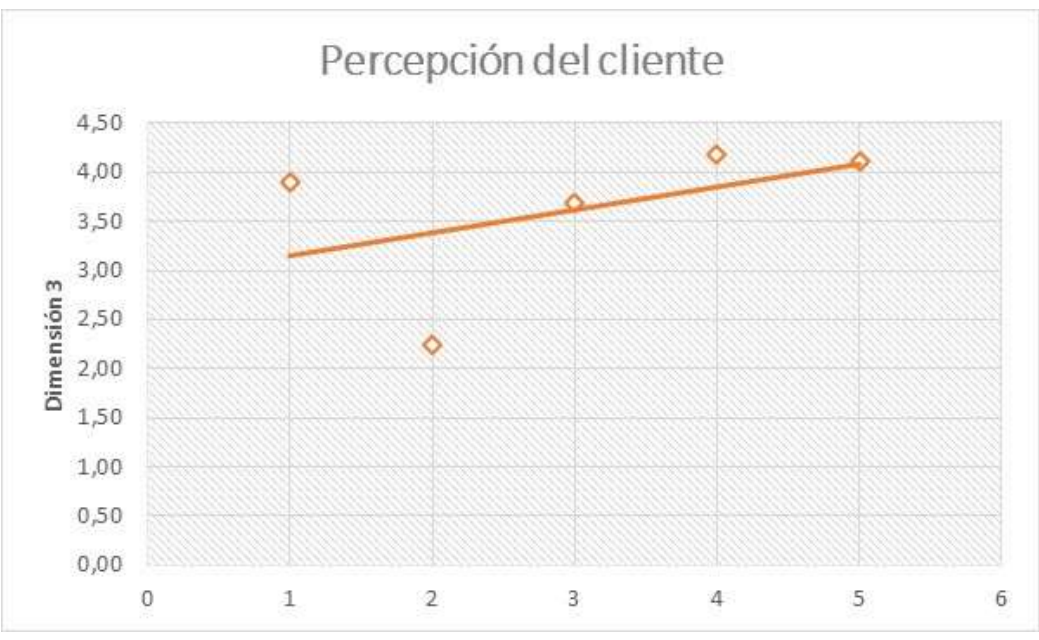

Fuente: Elaboración propia a partir de datos obtenidos por el software SPSS v.21

Basado en los datos obtenidos se puede mencionar que los atributos: 9 (área de servicio al cliente); 11 (área de administración y operativa); 13 (involucra a todas las áreas), obtuvieron parámetros de 2,16, 2,41 y 2,24 respectivamente, así pues, se detectaron tres problemas y sus causas principales: el primero es la atención al cliente poco servicial (causa: limitada 
capacitación y poca motivación al personal); el segundo excesiva demora en la atención al personal (causa: descoordinación en la atención al cliente); y por último una limitada comunicación en la organización (causa: inadecuada formación de trabajo en equipo).

Tabla 7. Toma de acciones correctivas.

\begin{tabular}{cccc}
\hline \multicolumn{4}{c}{ Toma de acciones correctivas y preventivas } \\
\hline Items & Descripción de problemas & Causas asignables & Efectos \\
& atención poco esmerada & $\begin{array}{c}\text { Limitada capacitación y } \\
\text { motivación del personal }\end{array}$ & Cliente insatisfecho \\
\hline & & Descoordinación en la & Baja productividad y \\
& Excesiva demora en la atención al cliente & atención oportuna al cliente & calidad del servicio \\
\hline & & Inadecuada formación de & Insatisfacción del \\
& Limitada comunicación & equipos de trabajo & personal \\
\hline
\end{tabular}

Fuente: Elaboración propia.

\section{DISCUSIÓN}

La atención al cliente es la base diferenciadora entre organizaciones, más aún, se debe tener en cuenta: el liderazgo, eficiencia, capital humano y cultura organizacional, por lo tanto, la capacitación del capital humano permite cambiar sistemáticamente su conducta y excelencia, incrementando su eficiencia organizacional. Los autores sugieren tres alternativas para minimizar el alto grado de insatisfacción al cliente, programa de capacitación, plan de motivación y formación de círculos de la calidad.

Tabla 8. Indicadores de servicio.

\begin{tabular}{|c|c|c|c|c|c|c|}
\hline \multicolumn{7}{|c|}{ INDICADORES DE SERVICIO } \\
\hline \multirow{2}{*}{$\begin{array}{l}\text { Código de } \\
\text { encuesta }\end{array}$} & \multirow{2}{*}{ Campo } & \multirow{2}{*}{ Área } & \multicolumn{2}{|c|}{ Párametros } & \multirow{2}{*}{ Problemas } & \multirow[b]{2}{*}{ Causas } \\
\hline & & & Actual & Esperado & & \\
\hline & & & & & & Limitada capacitación y \\
\hline & Atención del & & & & Atención poco & motivación del \\
\hline 9 & personal & Servicio al cliente & 2,16 & 5 & esmerada & personal \\
\hline 11 & $\begin{array}{l}\text { Rapidez en la } \\
\text { atención }\end{array}$ & $\begin{array}{c}\text { Administrativa y } \\
\text { operativa }\end{array}$ & 2,41 & 5 & $\begin{array}{l}\text { Excesiva demora en } \\
\text { la atención al cliente }\end{array}$ & $\begin{array}{c}\text { Descoordinación en la } \\
\text { atención oportuna al } \\
\text { cliente }\end{array}$ \\
\hline 13 & $\begin{array}{c}\text { Comunicación e } \\
\text { información }\end{array}$ & Todas & 2,24 & 5 & $\begin{array}{l}\text { Limitada } \\
\text { comunicación }\end{array}$ & $\begin{array}{l}\text { Inadecuada formación } \\
\text { de equipos de trabajo }\end{array}$ \\
\hline
\end{tabular}

Fuente: Elaboración propia. 
Nayul jodoy (2011) señala la necesidad de toda empresa en implantar programas de capacitación para fortalecer los conocimientos del personal en las tareas asignadas, más aún motivar que es la conjunción de los procesos fisiológicos y psicológicos en un momento dado para que el individuo se comporte en una determinada forma y logre los objetivos planteados por la organización.

Cada estrategia deberá cumplir con un objetivo principal, tiempo mínimo de seis meses, carga horaria diaria, y la temática debe variar según el objetivo propuesto, cada estrategia propuesta se basó en la Figura 1.

\section{CONCLUSIONES}

La competitividad se ha incrementado en las empresas turísticas, debido a que, el turismo es una actividad dinámica. La calidad se concibe como una poderosa arma competitiva que depende en gran medida del nivel de satisfacción que experimente el usuario.

En este caso, el sector de los restaurantes requiere de estrategias eficaces que puedan maximizar el grado de satisfacción de los clientes, por tanto, para alcanzar altos niveles de calidad en el servicio primero se mide el nivel de satisfacción y segundo se requiere de una filosofía empresarial en donde el primer objetivo sea la satisfacción al cliente.

El estudio realizado, en conjunto con la metodología aplicada, permitió evaluar la calidad de servicio que se ofrece en el restaurante Casa Blanca, evidenciándose la percepción que tienen los usuarios con respecto a las particularidades que conforman el servicio brindado.

Los instrumentos de recolección de datos aplicados revelaron altos niveles de viabilidad y fiabilidad. Para este análisis se utilizó el alfa de Cronbach con una media de $(0,96)$, así mismo el método de KMO (mayor a 0,9) y la prueba de esferidad de Bartlett con valores inferiores a 0,00 , por tanto, es aceptado el cuestionario utilizado.

La medición del nivel de satisfacción de los clientes, indicó que la calificación que le otorga el turista nacional y/o extranjero es igual a 3,45 (69\%), menor al 70\%, por lo tanto, a pesar de ser bueno este puntaje, no satisface los requerimientos de los organismos de control que esperan un porcentaje mayor al $80 \%$. 
Así mismo los atributos de la dimensión uno (2, 6 y 7); dimensión dos (1 y 3) y la dimensión tres (2) obtuvieron porcentajes por debajo de la media, así pues, son los peores atributos percibidos por los clientes.

En ese sentido, se hace necesario la puesta en marcha de un programa de mejora continua basado en un sistema de motivación y un plan de capacitación. Así mismo, implementar estrategias basadas en el modelo de gestión de la calidad Gap que incluya la misión, visión, el alcance, objetivos, políticas, responsabilidades y un sistema de seguimiento de acciones correctivas.

\section{REFERENCIAS BIBLIOGRÁFICAS}

Abud, D. (2009). Calidad total. Córdoba, AR: El Cid Editor | apuntes. Retrieved from http://www.ebrary.com

Aparecida Gouvêa, M., \& Oliveira, B. (2012). LA RELACIÓN ENTRE LA CALIDAD DE LOS SERVICIOS DE LAS AGENCIAS DE TURISMO Y LA MIGRACIÓN HACIA LA COMPRA POR INTERNET. Estudios y Perspectivas en Turismo, 21(3).

Árraga, B. M. V., \& Sánchez, V. M. (2012). Validez y confiabilidad de la Escala de Felicidad de Lima en adultos mayores venezolanos. Universitas Psychologica. Vol. 11(2), 2012. Bogotá, CO: Red Universitas Psychologica. Retrieved from http://www.ebrary.com

Bethencourt Cejas, M., Díaz Pérez, F. M., González Morales, M. O., \& Sánchez Pérez, J. (2005). La medición de la calidad de los servicios prestados por los destinos turísticos: el caso de las islas de La Palma. PASOS. Revista de Turismo y Patrimonio Cultural, 3(2).

Correia, P. G., Lucena, D. W. A., Madruga, L. C. F., de Menezes, P. D. L., \& de Sousa, P. P. R. (2012). Gestion de calidad del servicio de alimentos y bebidas: la importancia del manipulador de alimentos en la calidad del servicio hotelero de la ciudad de Joao Pessoa, Brasil. Estudios y Perspectivas en Turismo, 21(3), 763-778. 
Duque Oliva, E. J., Cervera Taulet, A., \& Rodríguez Romero, C. (2006). Estudio bibliométrico de los modelos de medición del concepto de calidad percibida del servicio en Internet. Innovar, 16(28), 223-243.

Duque, E. J. (2005). Revisión del concepto de calidad del servicio y sus modelos de medición. INNOVAR. Revista de Ciencias Administrativas y Sociales, 15() 64-80. Recuperado de http://www.redalyc.org/articulo.oa?id=81802505

Duque, O.; Edison J.; Chaparro P. y César R. (2012). Medición de la percepción de la calidad del servicio de educación por parte de los estudiantes de la UPTC Duitama. Criterio Libre, 10 (16), 159-192 ISSN 1900-0642

Fraiz Brea, J. A., Álvarez García, J., \& Del Río Rama, M. D. (2012). Motivaciones para implementar un sistema de gestión de la calidad. Análisis empírico en el sector turístico español. Cultur-Revista de Cultura e Turismo, Ilhéus, 6(1), 40-68.

García-Fernández, M. (2016). Influencia de la gestión de la calidad en los resultados de innovación a través de la gestión del conocimiento. Un estudio de casos. innovar, 26(61), 45-64.

García, J. Á., Brea, J. A. F. y del Río, M. D. L. C. (2015). GESTIÓN DE LA CALIDAD EN LAS TERMAS DE LA REGIÓN CENTRO DE PORTUGAL. CULTUR-Revista de Cultura e Turismo, 6(4), 86-112.

Gomes, R. W. y de Faria, R. C. (2012). Calidad de los sitios web en la percepción de los usuarios, una aplicación en los hoteles de Joao Pessoa, Paraíba Brasil. Estudios y Perspectivas de Turismo, 21(4), 925-944.

Gonçalves Gândara, J M; Fraiz Brea, J A; Manosso, F C; (2013). CALIDAD DE LA EXPERIENCIA EN LOS HOTELES TERMALES DE GALICIA, ESPAÑA. Un análisis a través de la reputación online. Estudios y Perspectivas en Turismo, 22() 492-525. Recuperado de http://4www.redalyc.org/articulo.oa?id=180726463007.

González, J. A. A. (2009). A practical application of statistical gap analysis in National Park Management in Costa Rica. COMITÉ EDITORIAL DIRECTOR: Agustín Santana Talavera, 7, 141. 
Holderbaum, B. S., de la Cruz, J. T. O., da Silva Lemos, A., \& Canaria-España, G. (2012). EL NIVEL DE CALIDAD DE NÚCLEOS TURÍSTICOS URBANIZADOS DEL TURISMO DE MASA Y LA PROPUESTA DE CIUDAD TURÍSTICA. Estudios y Perspectivas en Turismo, 21, 1336-1368.

Ibáñez, R. M. (2011). DIAGNÓSTICO DE LA CALIDAD Y COMPETITIVIDAD DEL SECTOR TURÍSTICO EN MÉXICO. Cuadernos de Turismo, 121-143. Recuperado de http://www.redalyc.org/articulo.oa?id=39821278007.

López, F. M. C. (2006). Dimensiones y medición de la calidad de servicio en empresas hoteleras. Bogotá, CO: Red Revista Colombiana de Marketing. Retrieved from http://www.ebrary.com.

Lloréns Montes, F. J. (1995). Un análisis de la importancia relativa que tienen las dimensiones de la calidad de servicio en la percepción del cliente. Cuadernos de Ciencias Económicas y Empresariales, (29), 35-45.

Mondo, T. S., \& Fiates, G. G. S. (2016). ATRIBUTOS DE LA CALIDAD DE SERVICIOS EN ATRACTIVOS TURÍSTICOS. Estudios y Perspectivas en Turismo, 25, 124-142.

Mendoza, A. J. A. (2009). Medición de la calidad del servicio. Córdoba, AR: El Cid Editor | apuntes. Retrieved from http://www.ebrary.com.

Moliner Velázquez, B; Gallarza, M G; Gil Saura, I; Fuentes Blasco, M; (2015). CAUSAS Y CONSECUENCIAS SOCIALES DE LA SATISFACCIÓN DE LOS CLIENTES CON HOTELES. Cuadernos de Turismo, 295-313. Recuperado de http://www.redalyc.org/articulo.oa?id=39841130013.

Morales, L. E. I., \& Medina, E. V. C. (2015). Aplicación del modelo Servperf en los centros de atención Telcel, Hermosillo: una medición de la calidad en el servicio. Contaduría y administración, 60(1), 229-260.

Najul Godoy, J; (2011). El capital humano en la atención al cliente y la calidad de servicio. Observatorio Laboral Revista Venezolana, (4) 23-35. Recuperado de http://www.redalyc.org/articulo.oa?id=219022148002 
Parasuraman, A., Zeithaml, V. A., \& Berry, L. L. (1985). A conceptual model of service quality and its implications for future research. the Journal of Marketing, 41-50.

Peralta Montecinos, J; (2006). Rol de las expectativas en el juicio de satisfacción y calidad percibida del servicio. Límite, 1() 195-214. Recuperado de http://www.redalyc.org/articulo.oa?id=83601409

Pozo, A. J. F. D. (2012). Procesos de gestión de calidad en hostelería y turismo: promoción turística local e información al visitante (UF0049). Málaga, ES: IC Editorial. Retrieved from http://www.ebrary.com

Raposo, W. G., \& de Faria Pereira, R. C. (2012). CALIDAD DE LOS SITIOS WEB EN LA PERCEPCIÓN DE LOS USUARIOS: Una aplicación en los hoteles de João Pessoa, Paraíba, Brasil. Estudios y Perspectivas en Turismo, 21(4), 925-945.

Ruiz Quesada, S. C., \& Parada Gutiérrez, Ó. (2016). PRINCIPALES VARIABLES PARA LA GESTIÓN DE LA CALIDAD DEL SERVICIO DE RECEPCIÓN DE TURISMO DE CRUCEROS. Cuadernos de Turismo, (38).

Sánchez, R. M.; Martínez, V; González, M G; Ramos, J y Peiró, J. M. (2009). Un análisis transnivel de las relaciones de la calidad de servicio y la confirmación de expectativas con la satisfacción de los usuarios. Psicothema, 21() 421-426. Recuperado de http://www.redalyc.org/articulo.oa?id=72711821013.

Sarmiento, J. R. (2017). La experiencia de la calidad de servicio online como antecedente de la satisfacción online: estudio empírico en los sitios web de viajes Investigaciones Turísticas. 2017, 13: 30-53. doi: 10.14198/INTURI2017. 13.02 URI: http://dx. doi. org/10.14198/INTURI2017. 13.02.

Shahin, A. (2004). SERVQUAL and Model of Service Quality Gaps: A Framework for Determining and Prioritizing Critical Factors in.

http://www.eltelegrafo.com.ec/noticias/economia/8/el-turismo-mundial-crecio-un-4-en-2016segun-la-omt DE: www.eltelegrafo.com.ec 
Vargas, Q. M. E., \& Aldana, D. V. L. (2011). Calidad y servicio: conceptos y herramientas (2a. ed.). Bogotá, CO: Ecoe Ediciones. Retrieved from http://www.ebrary.com.

Velázquez, B. M., Gallarza, M. G., Saura, I. G., \& Blasco, M. F. (2015). Causas y consecuencias sociales de la satisfacción de los clientes con hoteles. Cuadernos de Turismo, (36), 295-313.

Vera, M., J; Trujillo, L. A. (2009). El Papel de la Calidad del Servicio del Restaurante como Antecedente de la Lealtad del Cliente. Panorama Socioeconómico, 27() 16-30. Recuperado de http://www.redalyc.org/articulo.oa?id=39912023003

Yarimoglu, E. K. (2014). A review on dimensions of service quality models. Journal of Marketing Management, 2(2), 79-93.

Zamora, J., León, A., \& Andrades, I. (2009). Percepción de la calidad de los servicios proporcionados en una reserva nacional: el caso del Radal Siete Tazas. Santiago de Chile, CL: B - Facultad de Ciencias Empresariales Universidad de Talca. Retrieved from http://www.ebrary.com. 